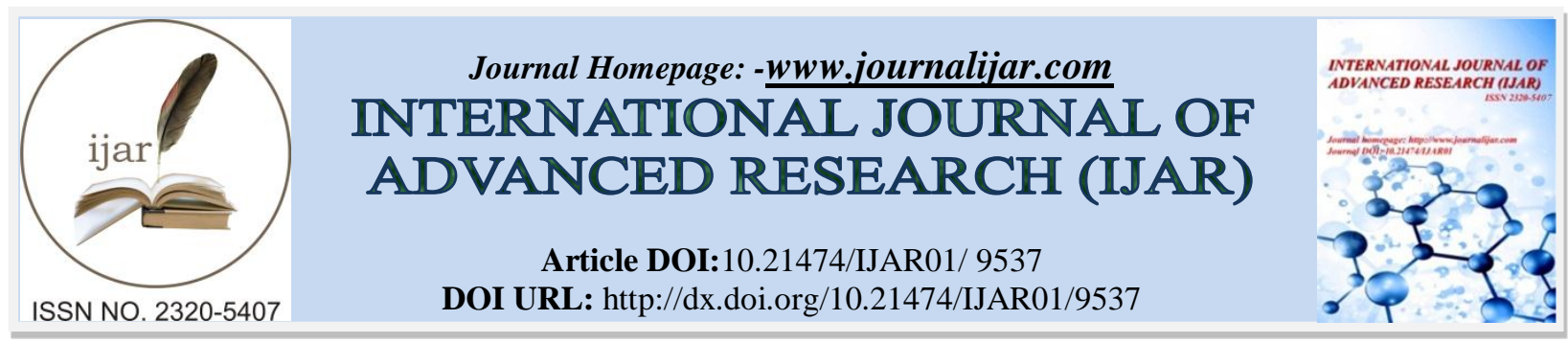

RESEARCH ARTICLE

\title{
LE LAMBEAU D'ORTICOCHEA REVISITE : A PROPOS DE 11 CAS. REVISITED ORTICOCHEA FLAP : ABOUT 11 CASES.
}

\section{J. Hafidi' ${ }^{12}$, M.A. Mokfi ${ }^{1}$, M. Raboune', S. El Mazouz'1, N. Gharib ${ }^{1}$, A. Abbassi' ${ }^{1}$, S. El Khloufi², M.L Bouchikhi² $^{2}$ M. Jiddane ${ }^{2}$ and Y. Bjijou' ${ }^{2}$.}

1. Service de chirurgie plastique et des brûlés, hôpital Ibn-Sina, Rabat.

2. Laboratoire d'anatomie, de microchirurgie et chirurgie expérimentale, faculté de médecine et de pharmacie de Rabat.

\section{Manuscript Info}

(.........................

Manuscript History

Received: 08 June 2019

Final Accepted: 10 July 2019

Published: August 2019

Key words:-

Revisited Orticochea flap, loss of substance, scalp.

\section{Abstract}

Introduction : Several loss of substance are located in the scalp and their treatment is subject to two requirements: reconstruction and a satisfactory aesthetic results

Material and method: From March 2016 to March 2019, 11 patients aged between 24 and 45, with a loss of substance of the scalp of 8 to $14 \mathrm{~cm}$, caused by electric burns by high voltage and by accidents, were treated with a revisited Orticochea flap.

Discussion : The reconstruction use a technique inspired by the Orticochea flap but the design and the implementation are simpler. It takes off the scalp very far and then fragments it into several flaps. The rotation, originality of our technique, is achieved by the help of back cuts on the temporal flap and the second flap and if necessary on the occipital flap. This technique has the advantage of leaving a minimum area of alopecia and can close a wide loss of substance. No skin graft were necessary and there were no complications in our cases.

Conclusion: This technique is satisfying, with good aesthetic results and a good sensitivity of the scalp.

Copy Right, IJAR, 2019,. All rights reserved.

\section{Introduction:-}

Le scalp est souvent, de part sa vulnérabilité et son exposition, le siège de pertes de substance parfois importantes, leur prise en charge dans un service de chirurgie plastique est soumise à deux impératifs : la reconstruction de cette perte de substance, et un résultat esthétique satisfaisant pour le patient [1]. Parmi les différents types de lambeau que l'on peut utiliser pour la couverture du scalp, le lambeau d'Orticochéa revisité nous semble être un lambeau de choix. Notre travail décrit une série de 11 patients ayant bénéficié de cette technique au sein du service de chirurgie plastique de l'hôpital Ibn-Sina de Rabat.

\section{Matériel et méthode:-}

De mars 2016 à mars 2019, 11 patients âgés de 24 à 45 ans, porteurs de perte de substance du scalp voisines de la région tonsurale ont été pris en charge dans notre service. Sur les 11 patients, nous distinguons 10 hommes et 1 femme. Les étiologies de ces pertes de substance sont les brûlures électriques du scalp dans la majorité des cas avec 8 patients, et les accidents de la voie publique dans 3 cas. Le diamètre des pertes de substance du scalp varie de 8 à

Corresponding Author:-J. Hafidi.

Address:-Service de chirurgie plastique et des brûlés, hôpital Ibn-Sina, Rabat. 
$14 \mathrm{~cm}$. Les malades brulés ont été pris en charge initialement en réanimation, une fois le pronostic vital mis hors de danger, ils ont bénéficié de soins locaux et de pansements gras changés tous les jours. La prise en charge chirurgicale proprement dite ne s'est faite qu'un mois plus tard. Les malades victimes d'accidents de la voie publique ont été pris en charge par nos soins une semaine plus tard, après qu'aucune urgence neurologique n'ait été décelée et que le pronostic vital ait été mis hors de cause. Aucun cas d'ostéite n'est à déplorer. 1 patient a souffert de troubles neurologiques à type de paresthésies au niveau des extrémités accompagnées de céphalées. Le scanner pour ces 2 malades est revenu normal. Les patients sont opérés sous anesthésie générale. Nous réalisons un parage des berges de la perte de substance et de l'os (figure 1,2). La technique utilisée est inspirée de celle d'Orticochéa. Tout le scalp est mobilisé en sous-galéal, puis quatre lambeaux: deux temporaux, un frontal et un occipital sont levés, leurs bases étant égales au diamètre de la perte de substance et incluant l'axe vasculaire principal de la région (figure 3-5). Ils bénéficient d'un mouvement d'avancement - rotation vers la perte de substance (figure 7,8), l'avancement pur étant toujours insuffisant. Cette rotation, originalité de cette géométrie, rend ces lambeaux d'une efficacité extrême pour couvrir de tels déficits; elle est possible grâce à des contre-incisions ou back-cuts (figure 6) judicieusement placées au niveau du premier lambeau temporal puis du second et si nécessaire du lambeau occipital mais jamais aux dépens du lambeau frontal.

\section{Résultats:-}

Une couverture complète de la perte de substance est obtenue par cette technique (figure 9). Aucun malade n'a nécessité de greffe de peau, la cicatrisation des back-cuts a pris approximativement 5 semaines, des pansements gras changés tous les jours accélérant le processus de cicatrisation. Aucune complication n'a été déplorée, tous nos patients ont été satisfaits du résultat esthétique, la repousse des cheveux s'est faite de façon normale (figure 10). La sensibilité du scalp est redevenue quasi-normale en quelques semaines.

\section{Discussion:-}

Les principaux lambeaux locaux utilisés pour la couverture des pertes de substance du scalp sont les lambeaux conventionnels et les lambeaux vasculaires. Les lambeaux conventionnels comprennent les lambeaux d'avancement, de rotation-glissement type lambeaux d'IMRE ou deux lambeaux type Converse ou trois lambeaux type Gillies, citons aussi les lambeaux bipédiculés ; les lambeaux à axe oblique antéro-postérieur frontal en avant et occipital controlatéral en arrière ; les lambeaux à axe longitudinal sur les vaisseaux frontaux en avant, occipital homolatéral en arrière; les lambeaux transversaux pédiculés sur les deux axes temporaux superficiels (et/ou auriculaires postérieurs) et enfin, les lambeaux multiples d'Orticochéa pour les grandes pertes de substance. Les lambeaux vasculaires incluent les lambeaux axiaux temporaux transposés vers l'avant ou vers l'arrière d'Onizuka sur les branches temporo-pariétales pour des pertes de substance temporale ou occipitale ; les lambeaux axiaux occipitaux de Thompson ou de Lequang et les lambeaux axiaux anastomotiques surtout temporaux comme le lambeau bifolié de Wilson [2,3].

Le lambeau d'Orticochéa revisité décolle le scalp très loin puis le fragmente en plusieurs lambeaux (3 ou 4), au mieux axés sur un pédicule. La striation de la galéa de ces lambeaux, associée à leur transposition, leur glissement et à leur rotation, permet la couverture d'importantes pertes de substance (de 100 à $150 \mathrm{~cm} 2$ ) des régions : frontale, occipitale, pariéto-occipitale ou même du vertex. La rotation, originalité de notre technique, est réalisée grâce à des contre-incisions (back-cuts) placées au niveau du premier lambeau temporal puis du second et si nécessaire du lambeau occipital mais jamais aux dépens du lambeau frontal. Cette technique a l'avantage de laisser un minimum de zone alopécique tout en permettant la fermeture de pertes de substance très importantes. Cependant, elle a surtout l'inconvénient de morceler complètement le scalp restant [4,5].

\section{Conclusion:-}

Le lambeau d'Orticochéa revisité est un lambeau de choix dans la prise en charge des pertes de substance du scalp, notamment dans les grandes perte de substance que l'on doit gérer au décours de brûlures électriques par haut voltage ou lors d'accidents de la voie publique. Cette technique, dont ont bénéficié les patients que nous avons pris en charge a donné de très bons résultats à la fois fonctionnels et esthétiques. 


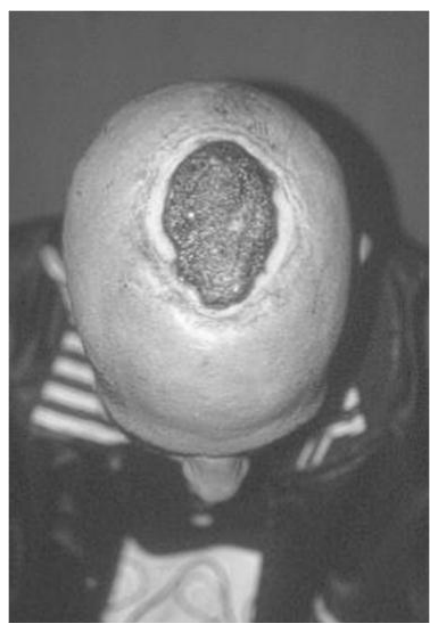

Fig 1:-patient présentant une PDS du scalp par brûlure électrique

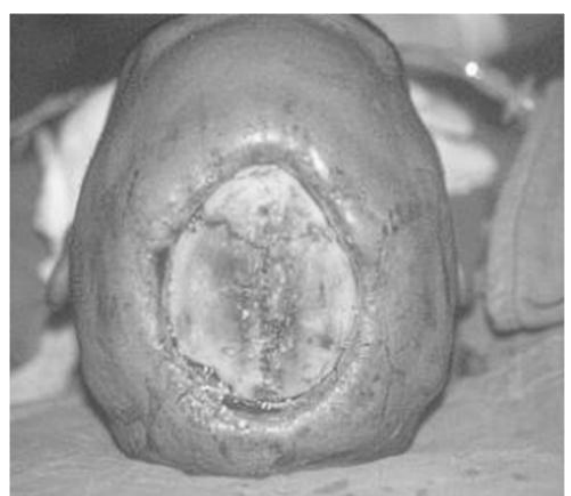

Fig 2:-Parage de la zone nécrosée

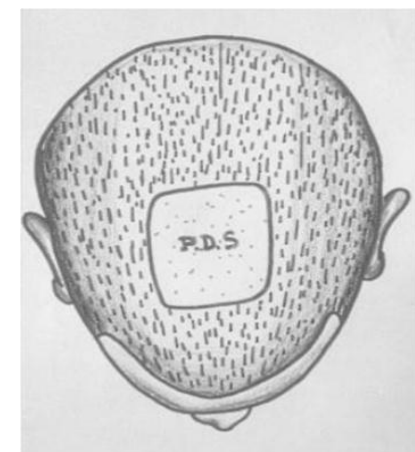

Fig 3:-Schématisation de la PDS 


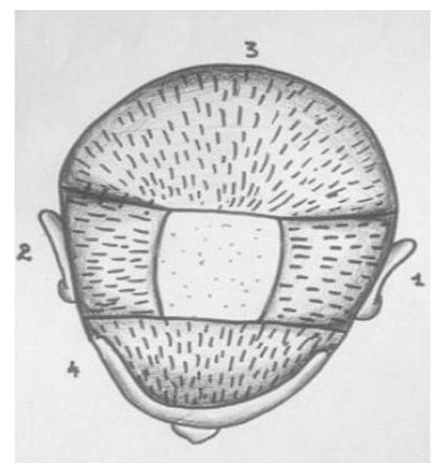

Fig 4:-Tracé des incisions

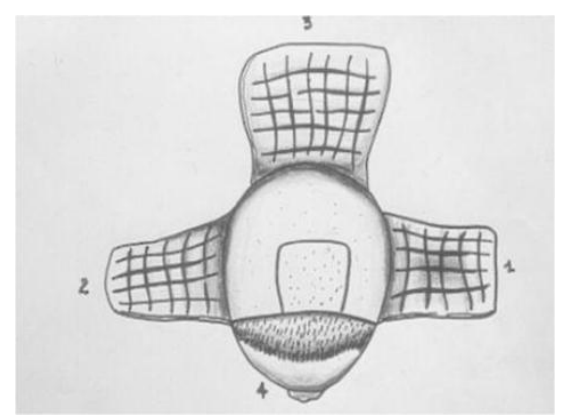

Fig 5:-Levée des lambeaux

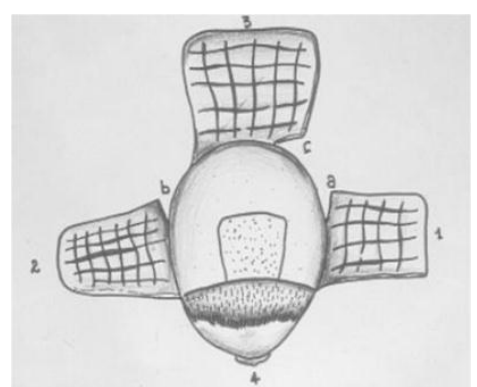

Fig 6:-Back cut sur les lambeaux temporaux et sur le lambeau occipital

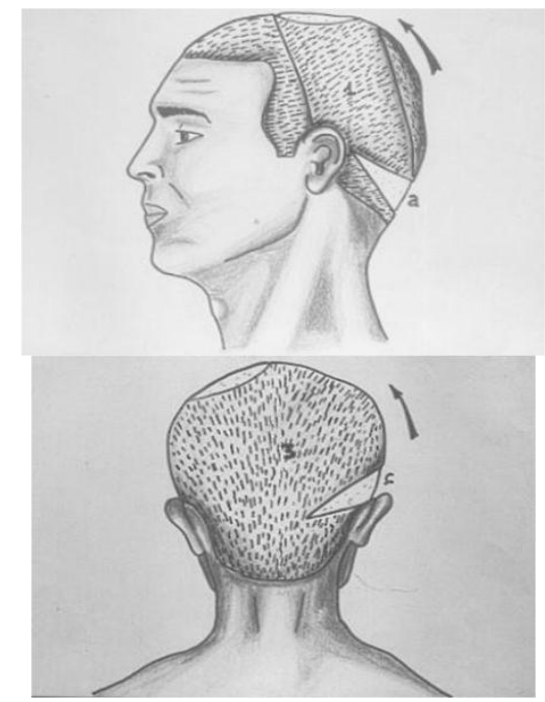

Fig 7 et 8:-Avancement - rotation des lambeaux 


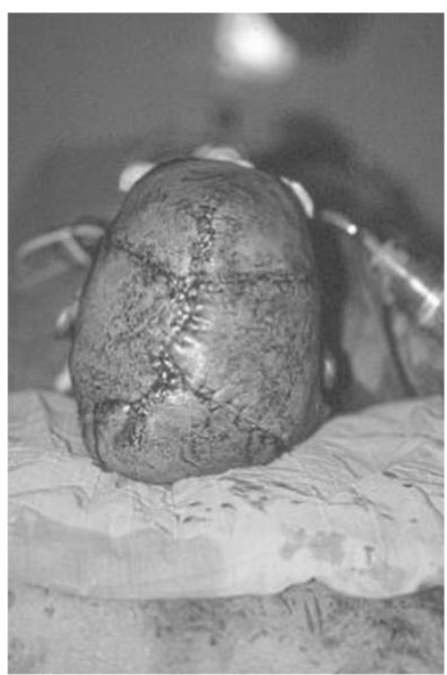

Fig 9:-Aspect après mise en place et suture des lambeaux

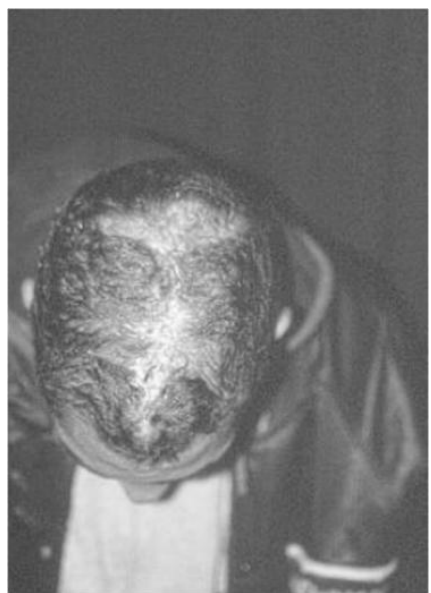

Fig 10:-Résultat à 6 mois

\section{Bibliographie:-}

1. Chassagne J.F., Brice M., Maxant P., Flot F., Ginh Don G., Stricker M.: Evaluation des idées dans les techniques de réparation du scalp. Ann. Chir. Plast., 31: 325-35, 1996.

2. Lafautrie P.: Progrès récents dans la chirurgie du cuir chevelu. Thèse de Médecine, Paris VII, 1987.

3. P. Banzet, JM Servant. : Chirurgie plastique, reconstructrice et esthétique, II : 118-120

4. Brice M., Maxant F., Flot F., Dinh Dean G., Stricker M. Evolution des idées dans les techniques de réparation du scalp. Ann Chir Plast Esthét. 31:325-335.

5. Perez M. Reconstruction des pertes de substance du cuir chevelu. Thèse; Rennes: 1983. 